\title{
Perfil soroepidemiológico da hepatite B em localidades ribeirinhas do rio Madeira, em Porto Velho, Estado de Rondônia, Brasil
}

Serumepidemiological profile of hepatitis B in riverine localities of the Madeira River, Porto Velho, Rondônia State, Brazil

Perfil seroepidemiológico de la hepatitis B en localidades ribereñas del río Madeira, en Porto Velho, Estado de Rondônia, Brasil

Adriane Cristine Barbosa e Silva

Centro de Pesquisa em Medicina Tropical, Porto Velho, Rondônia, Brasil

Luan Felipo Botelho Souza

Fundação Oswaldo Cruz de Rondônia, Porto Velho, Rondônia, Brasil

Tony Hiroshi Katsuragawa

Fundação Oswaldo Cruz de Rondônia, Porto Velho, Rondônia, Brasil

\author{
Alzemar Alves de Lima \\ Centro de Pesquisa em Medicina Tropical, Porto Velho, Rondônia, Brasil \\ Deusilene Souza Vieira \\ Fundação Oswaldo Cruz de Rondônia, Porto Velho, Rondônia, Brasil \\ Juan Miguel Villalobos Salcedo \\ Fundação Oswaldo Cruz de Rondônia, Porto Velho, Rondônia, Brasil
}

\section{RESUMO}

Em duas localidades ribeirinhas do Município de Porto Velho, Estado de Rondônia, Brasil, foi realizado um estudo observacional, de corte transversal, para avaliar o perfil soroepidemiológico do vírus da hepatite B (VHB) e possíveis fatores de risco na transmissão. Foram analisadas 660 amostras, por meio de exames sorológicos pelo método ELISA, para o VHB (anti-HBc total, anti-HBs e HBsAg). Os resultados mostraram que 50,7\% (335) das amostras apresentaram positividade para algum tipo de marcador sorológico. $\mathrm{OHBsAg}$ foi reagente em $12(1,8 \%)$ amostras, sendo 11 (91,6\%) oriundas de indivíduos do sexo masculino. O anti-HBc total reagente ocorreu em 212 (32,1\%) das amostras e distribuído em todas as faixas etárias, com exceção da faixa de 11 a 16 anos de idade. $\mathrm{O}$ anti-HBs reagente esteve presente em $239(36,2 \%)$ das amostras, com as maiores prevalências nos grupos etários a partir dos 21 anos de idade. $O$ estudo permitiu observar que as localidades analisadas possuem baixa endemicidade para portadores crônicos, entretanto observou-se uma alta prevalência para a infecção. A porcentagem de indivíduos suscetíveis foi de 49,2\% (325), sendo maior entre indivíduos entre 6 e 15 anos de idade. Os fatores de transmissão, como extrações dentárias, cirurgias e compartilhamento de materiais de higiene pessoal estão possivelmente associados à infecção pelo VHB na região analisada. Além disso, a baixa porcentagem do marcador anti-HBs direciona para uma falha na cobertura vacinal na população avaliada no presente estudo.

Palavras-chave: Vírus da Hepatite B; Prevalência; População Rural; Cobertura Vacinal.

\section{INTRODUÇÃO}

O vírus da hepatite B (VHB) possui distribuição mundial e constitui um importante problema de saúde pública. Estima-se que mais de 2 bilhões de pessoas apresentam evidências sorológicas de infecção passada ou presente pelo VHB e que 350 milhões são portadores crônicos ${ }^{1}$. Cerca de 0,5 a 1,2 milhões de pessoas morrem anualmente em virtude de complicações ocasionadas por esse vírus².

A epidemiologia da infecção pelo VHB tradicionalmente tem sido descrita em três categorias de endemicidade: alta, intermediária e baixa, dependendo da proporção da população soropositiva para $\mathrm{HBsAg}^{3}$. Aproximadamente $45 \%$ da população mundial vive em áreas consideradas altamente endêmicas para 0 vírus (8\% da população são $\mathrm{HBs} A g$ positivas), $43 \%$ vive em áreas de endemicidade intermediária para o VHB (2-7\% da população são $\mathrm{HBsAg}$ positivas) e $12 \%$ vive em áreas de baixa endemicidade ( $>2 \%$ da população são HBsAg positivas)'. 
No Brasil, estima-se que aproximadamente 15\% da população apresenta evidências sorológicas de contato com o vírus e os casos crônicos devem corresponder a $1 \%{ }^{4}$. Em relação ao Estado de Rondônia, os dados mais recentes do Ministério da Saúde e do Programa Nacional das Hepatites Virais mostram que no ano de 2010 foram notificados 345 casos de hepatite $B^{5}$. No mesmo ano, o Instituto Brasileiro de Geografia e Estatística $^{6}$ registrou uma população total de 1.562.409 habitantes. Isso permite estimar a taxa de detecção do VHB em Rondônia em 22,1 (por 100.000 habitantes), sendo a segunda maior taxa da Região Norte no período.

Embora Rondônia seja classificado como área hiperendêmica para as hepatites virais, os dados de prevalência são escassos e fragmentados, coletados por alguns poucos estudos que devem ser complementados. Existem vários fatores que podem contribuir para determinar esta endemicidade: (i) primeiramente, o desconhecimento da verdadeira magnitude local do problema, pois estudos realizados em Estados vizinhos, como o Acre e o Amazonas, não mostram os mesmos fatores epidemiológicos do Estado objeto do presente estudo; (ii) outro fator é a falta de um estudo amplo de base populacional que possa substanciar essa questão. Nesse sentido, este trabalho visa contribuir para o esclarecimento de alguns destes interrogantes, por meio da análise da prevalência da infecção pelo VHB em comunidades ribeirinhas próximas a grandes empreendimentos em andamento no rio Madeira, que atraíram milhares de trabalhadores diretos e indiretos. Como questão de saúde pública, o conhecimento da epidemiologia e prevalência da doença é fundamental para nortear as políticas públicas de saúde frente às grandes obras civis na Amazônia.

\section{MATERIAIS E MÉTODOS}

\section{ÁREA DE ESTUDO}

Trata-se de um inquérito soroepidemiológico de corte transversal, analítico-observacional, realizado em duas comunidades ribeirinhas do Município de Porto Velho, Estado de Rondônia, denominadas Cachoeira do Teotônio e Vila Amazonas, localizadas às margens direita e esquerda do rio Madeira, com população de 522 e 253 habitantes, respectivamente (Figura 1). Ambas as localidades foram diretamente atingidas pela construção da usina hidrelétrica (UHE) de Santo Antônio, iniciada em 2008. Essas localidades iá foram objeto de estudos anteriores, sobre as quais foi feita uma descrição detalhada $7,8,9$.

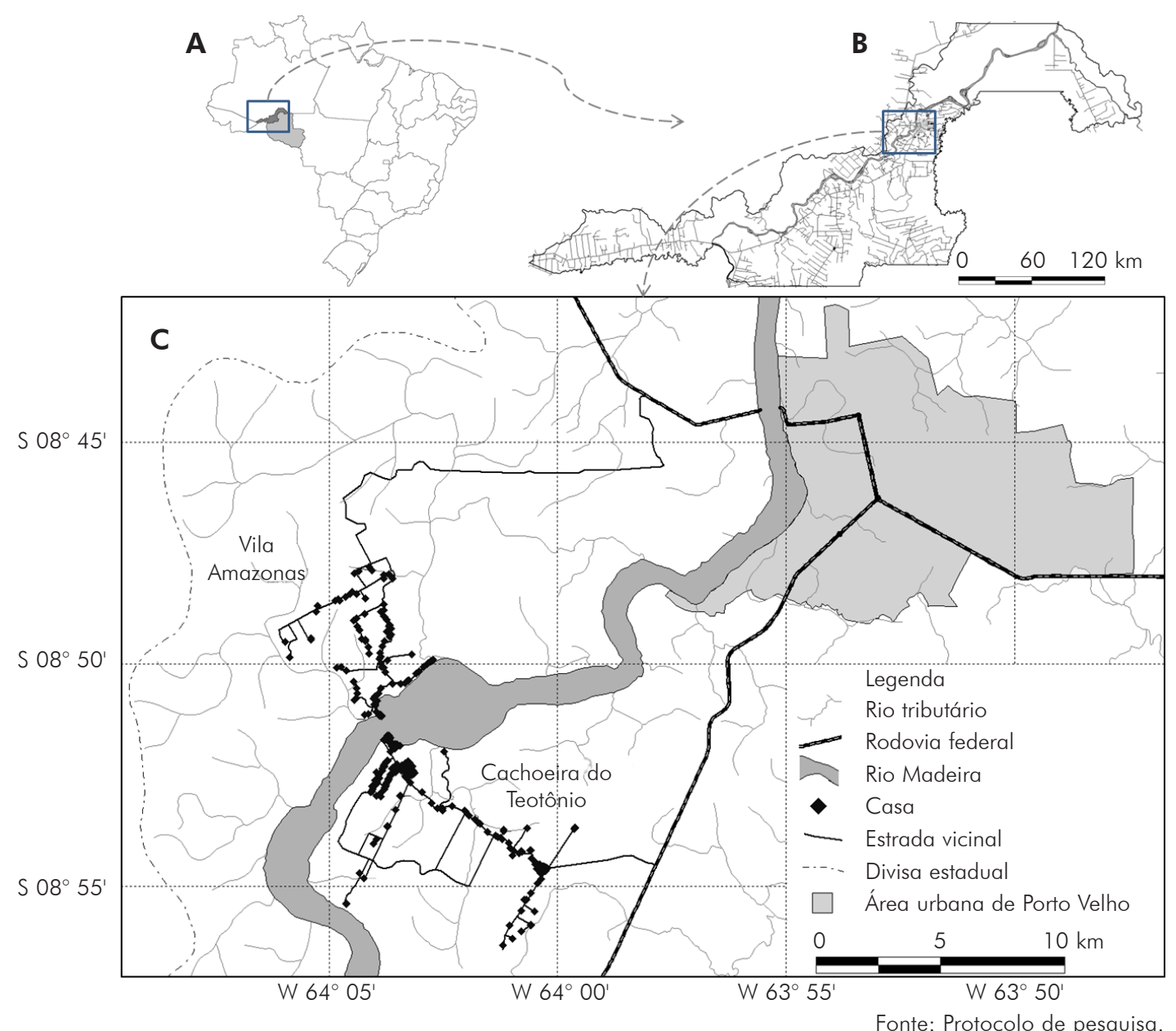

A: Localização no Estado de Rondônia; B: Localização no Município de Porto Velho; C: A Vila Amazonas está localizada à margem esquerda do rio Madeira e a localidade de Cachoeira do Teotônio à margem direita.

Figura 1 - Mapa da área de estudo 


\section{INFORMAÇÕES EPIDEMIOLÓGICAS}

No momento da coleta das amostras biológicas foram aplicados dois documentos epidemiológicos, ambos estruturados, sendo um Questionário Epidemiológico Familiar (QEF) e outro denominado Questionário Epidemiológico Individual (QEI), nos quais foram englobadas diversas variáveis como: identificação, sexo, idade, tempo de moradia na região, condições sociais de moradia, histórico de vacinação para hepatite B, histórico de infecção pelo VHB na família, uso de preservativo em relações sexuais, compartilhamento de utensílios de higiene pessoal, injeção por curioso (aquele que relatou experiência de droga ilícita injetável uma única vez), transfusão sanguínea, dentre outras variáveis. Para permitir análise epidemiológica de vacinas, os participantes foram divididos por gênero e grupos etários.

\section{AMOSTRAS BIOLÓGICAS E ASPECTOS ÉTICOS}

As amostras biológicas utilizadas no presente estudo foram constituídas de sangue periférico dos participantes, obtidas após seu prévio consentimento, coletadas no período de abril de 2008 a agosto de 2010. Logo após a coleta, o soro foi separado dos elementos figurados do sangue por centrifugação e enviado para análise de marcadores sorológicos do VHB. Este estudo foi aprovado pelo Comitê de Ética em Pesquisa envolvendo seres humanos do Centro de Pesquisa em Medicina Tropical (CEP-CEPEM), possuindo os registros de números 070/2008 e 047/2011.

\section{MARCADORES SOROLÓGICOS DO VHB}

Foram determinados os seguintes marcadores sorológicos para a hepatite B: $\mathrm{HBsAg}$, anti-HBsAg e anti-HBc total, empregando-se os testes imunoenzimáticos - ELISA (DiaSorin S.A.: anti-HBc total: ETI-AB-Corek PLUS; HBsAg: ETI-MAK-4; anti-HBc 16 IgM: ETI-CORE-IGMK PLUS, Madrid, Espanha; Organon Teknika: anti-HBs, Boxtel, Holanda).

\section{ANÁLISES ESTATÍSTICAS}

Para a análise estatística, foram utilizados os softwares Epi Info ${ }^{T M}$ versão 3.5.2, e GraphPad Prism ${ }^{\circledR}$ versão 5.0 para teste $\dagger$ de Student e Mann Whitney, com valor de significância $p<0,05$. Para análise de ocorrência das variáveis categóricas, foram utilizadas tabelas de contingência $2 \times 2$, aplicando-se o teste qui-quadrado com correção de Yates para comparar as proporções, quando aplicável.

\section{RESULTADOS}

PERFIL DEMOGRÁFICO E PREVALÊNCIA DOS MARCADORES SOROLÓGICOS DO VHB

Todos os habitantes das duas localidades foram convidados a colaborar. Participaram da pesquisa 660 indivíduos (660/775, 85,2\%) sendo 212 (32,1\%) de Vila Amazonas e $448(67,9 \%)$ residentes em Cachoeira de Teotônio (Tabela 1). A idade variou entre 8 meses e 88 anos $(\bar{\chi}=31,6$ anos; $\sigma=20,0)$. A distribuição da população estudada, de acordo com o sexo e grupo etário, em Vila Amazonas e Cachoeira do Teotônio, apresentou diferença estatisticamente significante $(p<0,05)$. Para o marcador HBsAg, dos 12 resultados positivos, apenas um indivíduo foi do sexo feminino $(8,3 \%)$. O anti-HBc total foi positivo para $212(32,1 \%)$ indivíduos e o anti-HBs foi positivo para $239(36,2 \%)$ indivíduos.

Tabela 1 - Distribuição por grupo etário, sexo e resultado reagente para os marcadores sorológicos do VHB das localidades de Vila Amazonas e Cachoeira do Teotônio, Porto Velho, Estado de Rondônia, Brasil, $2008-2010$

\begin{tabular}{|c|c|c|c|c|c|c|c|c|c|}
\hline \multirow{2}{*}{ Localidade } & \multirow{2}{*}{ Grupo etário* } & \multicolumn{2}{|c|}{ Número de participantes } & \multicolumn{2}{|c|}{ Anti-HBc total } & \multicolumn{2}{|c|}{$\mathrm{HBsAg}$} & \multicolumn{2}{|c|}{ Anti-HBs } \\
\hline & & Masculino & Feminino & Masculino & Feminino & Masculino & Feminino & Masculino & Feminino \\
\hline \multirow{10}{*}{ Vila Amazonas } & $0-6$ & 9 & 5 & 1 & - & - & - & 2 & 1 \\
\hline & $6-11$ & 9 & 19 & - & - & - & - & 2 & 8 \\
\hline & $11-16$ & 13 & 11 & - & - & - & - & 5 & 2 \\
\hline & $16-21$ & 10 & 5 & 2 & - & 1 & - & 1 & 2 \\
\hline & $21-31$ & 11 & 9 & 1 & 2 & 1 & - & 1 & 5 \\
\hline & $31-41$ & 18 & 10 & 7 & 2 & 1 & - & 6 & 3 \\
\hline & $41-51$ & 24 & 13 & 10 & 7 & - & - & 9 & 4 \\
\hline & $51-61$ & 19 & 6 & 11 & 5 & 2 & - & 6 & 4 \\
\hline & $\geq 61$ & 16 & 5 & 12 & 2 & - & - & 4 & 2 \\
\hline & Total & 129 & 83 & 44 & 18 & 5 & - & 36 & 31 \\
\hline \multirow{10}{*}{ Cachoeira do Teotônio } & $0-6$ & 7 & 15 & - & - & - & - & - & 4 \\
\hline & $6-11$ & 31 & 20 & 1 & - & - & - & 6 & 5 \\
\hline & $11-16$ & 38 & 32 & - & - & - & - & 14 & 5 \\
\hline & $16-21$ & 18 & 23 & - & 1 & - & - & 6 & 11 \\
\hline & $21-31$ & 34 & 27 & 10 & 4 & 3 & - & 11 & 11 \\
\hline & $31-41$ & 32 & 32 & 20 & 15 & 1 & - & 13 & 10 \\
\hline & $41-51$ & 32 & 24 & 28 & 16 & 1 & 1 & 15 & 12 \\
\hline & $51-61$ & 27 & 10 & 20 & 7 & 1 & - & 17 & 9 \\
\hline & $\geq 61$ & 32 & 14 & 21 & 7 & - & - & 16 & 7 \\
\hline & Total & 251 & 197 & 100 & 50 & 6 & 1 & 98 & 74 \\
\hline
\end{tabular}

* Na linha do grupo etário, a primeira idade é completa e a segunda incompleta.

Sinal convencional utilizado: - Dado numérico igual a zero não resultante de arredondamento. 
Foram classificados sorologicamente os resultados dos marcadores sorológicos e as respectivas combinações de cada participante (Tabela 2). Seguindo essa classificação, somaram-se 12 (1,8\%) portadores crônicos, sendo 11 $(91,6 \%)$ do sexo masculino, distribuídos entre 16 e 60 anos de idade, com maior ocorrência no grupo etário 21-31 anos (33,3\%), como visto na figura 2 .

No geral, o anti-HBc total esteve presente em praticamente todas as faixas etárias, com exceção do grupo etário 11-16 anos. Os indivíduos do sexo masculino do grupo etário 41-51 anos foram mais predominantes em relação à presença do marcador de infecção.

Em relação ao anti-HBs, anticorpo que esteve presente em todas as faixas etárias, houve predomínio entre os indivíduos no grupo etário 41-51 anos, embora este marcador esteja, em sua maioria, associado ao anti-HBc total.
Se desconsiderados os resultados $(n=8) \mathrm{da}$ classificação sorológica indeterminada (Tabela 2), os portadores crônicos apresentaram a menor prevalência $(1,8 \%)$, enquanto que os suscetíveis foram os que apresentaram maior prevalência $(49,2 \%)$.

Dessa forma, na distribuição por grupo etário, observa-se ainda que o número de suscetíveis continua elevado (Tabela 3). As maiores frequências de vacinados foram observadas nos grupos etários de 0-31 anos.

\section{ANÁLISE UNIVARIADA}

A análise univariada entre gênero e idade com - marcador sorológico $\mathrm{HBsAg}$ revelou que os indivíduos do sexo masculino e com idade superior a 20 anos estão mais suscetíveis a tornarem-se portadores crônicos do VHB (Tabela 4). Também houve associação positiva para $\bigcirc$ marcador anti-HBc total $(p<0,001)$.

Tabela 2 - Resultados dos marcadores sorológicos dos 660 indivíduos submetidos à sorologia por ELISA e suas respectivas classificações sorológicas, Porto Velho, Estado de Rondônia, Brasil, 2008-2010

\begin{tabular}{llrc}
\hline Marcadores sorológicos reagentes & Classificação sorológica & Total & $\%$ \\
\hline $\mathrm{HBsAg}+$ anti-HBc total & Portadores crônicos & 12 & 1,8 \\
Anti-HBs & Vacinados & 118 & 17,9 \\
Anti-HBc total & Isolados & 76 & 11,5 \\
Anti-HBc total + anti-HBs & Imunes & 121 & 18,3 \\
Anti-HBc total + anti-HBs $+\mathrm{HBsAg}$ & Indeterminados & 8 & 1,2 \\
Nenhum marcador reagente & Suscetíveis & 325 & 49,2 \\
\hline Total & & 660 & 100,0 \\
\hline N: Número de participantes. Critérios para classificação sorológica ${ }^{10}$. & Fonte: Protocolo de pesquisa.
\end{tabular}

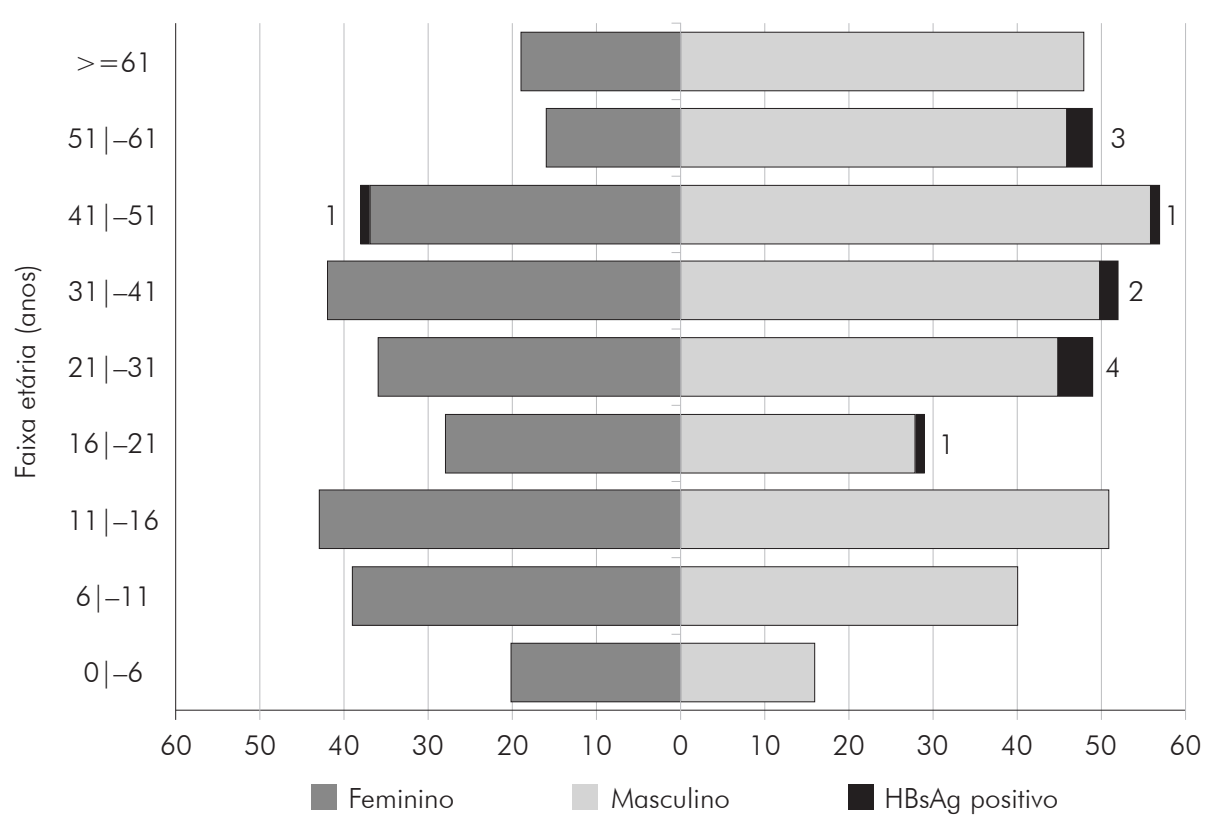

Fonte: Protocolo de pesquisa.

Figura 2 - Distribuição do marcador HBsAg positivo, por gênero e faixa etária do total da população, Porto Velho, Estado de Rondônia, Brasil, 2008-2010 
Tabela 3 - Distribuição do perfil sorológico, de acordo com a idade, em 652 indivíduos*, Porto Velho, Estado de Rondônia, Brasil, 2008-2010

\begin{tabular}{|c|c|c|c|c|c|c|c|c|c|c|c|c|}
\hline \multirow{3}{*}{ Idade (anos) } & \multicolumn{10}{|c|}{ Perfil sorológico } & \multirow{2}{*}{\multicolumn{2}{|c|}{ Total }} \\
\hline & \multicolumn{2}{|c|}{ Crônico } & \multicolumn{2}{|c|}{ Imune } & \multicolumn{2}{|c|}{ Vacinado } & \multicolumn{2}{|c|}{ Isolado ${ }^{\dagger}$} & \multicolumn{2}{|c|}{ Suscetível } & & \\
\hline & $\mathrm{N}$ & $\%$ & N & $\%$ & N & $\%$ & $\mathrm{~N}$ & $\%$ & $N$ & $\%$ & $\mathrm{~N}$ & $\%$ \\
\hline $0-6$ & - & - & 1 & 0,2 & 6 & 0,9 & - & - & 28 & 4,2 & 35 & 5,4 \\
\hline $6-11$ & - & - & 1 & 0,2 & 20 & 3,0 & - & - & 56 & 8,5 & 77 & 11,8 \\
\hline $11-16$ & - & - & - & - & 26 & 3,9 & - & - & 66 & 10,0 & 92 & 14,1 \\
\hline $16-21$ & 1 & 0,2 & 1 & 0,2 & 19 & 2,9 & 1 & 0,2 & 34 & 5,2 & 56 & 8,6 \\
\hline $21-31$ & 4 & 0,6 & 11 & 1,7 & 17 & 2,6 & 2 & 0,3 & 47 & 7,1 & 81 & 12,4 \\
\hline $31-41$ & 2 & 0,3 & 27 & 4,1 & 5 & 0,8 & 14 & 2,1 & 43 & 6,5 & 91 & 14,0 \\
\hline $41-51$ & 2 & 0,3 & 31 & 4,7 & 9 & 1,4 & 26 & 3,9 & 23 & 3,5 & 91 & 14,0 \\
\hline $51-61$ & 3 & 0,5 & 28 & 4,2 & 8 & 1,2 & 12 & 1,8 & 11 & 1,7 & 62 & 9,5 \\
\hline$\geq 61$ & - & - & 21 & 3,2 & 8 & 1,2 & 21 & 3,2 & 17 & 2,6 & 67 & 10,3 \\
\hline Total & 12 & 1,8 & 121 & 18,4 & 118 & 17,9 & 76 & 11,5 & 325 & 47,6 & $652^{*}$ & 100,0 \\
\hline
\end{tabular}

* Excluíram-se os indivíduos classificados como indeterminados (8/660); N: Número de indivíduos na faixa etária; ${ }^{\dagger}$ Anti-HBc total positivo isoladamente. Sinal convencional utilizado: - Dado numérico igual a zero não resultante de arredondamento.

Tabela 4 - Análise univariada de possíveis associações entre as variáveis sexo e idade com a sorologia dos marcadores sorológicos ABsAg e anti-HBc total, Porto Velho, Estado de Rondônia, Brasil, 2008-2010

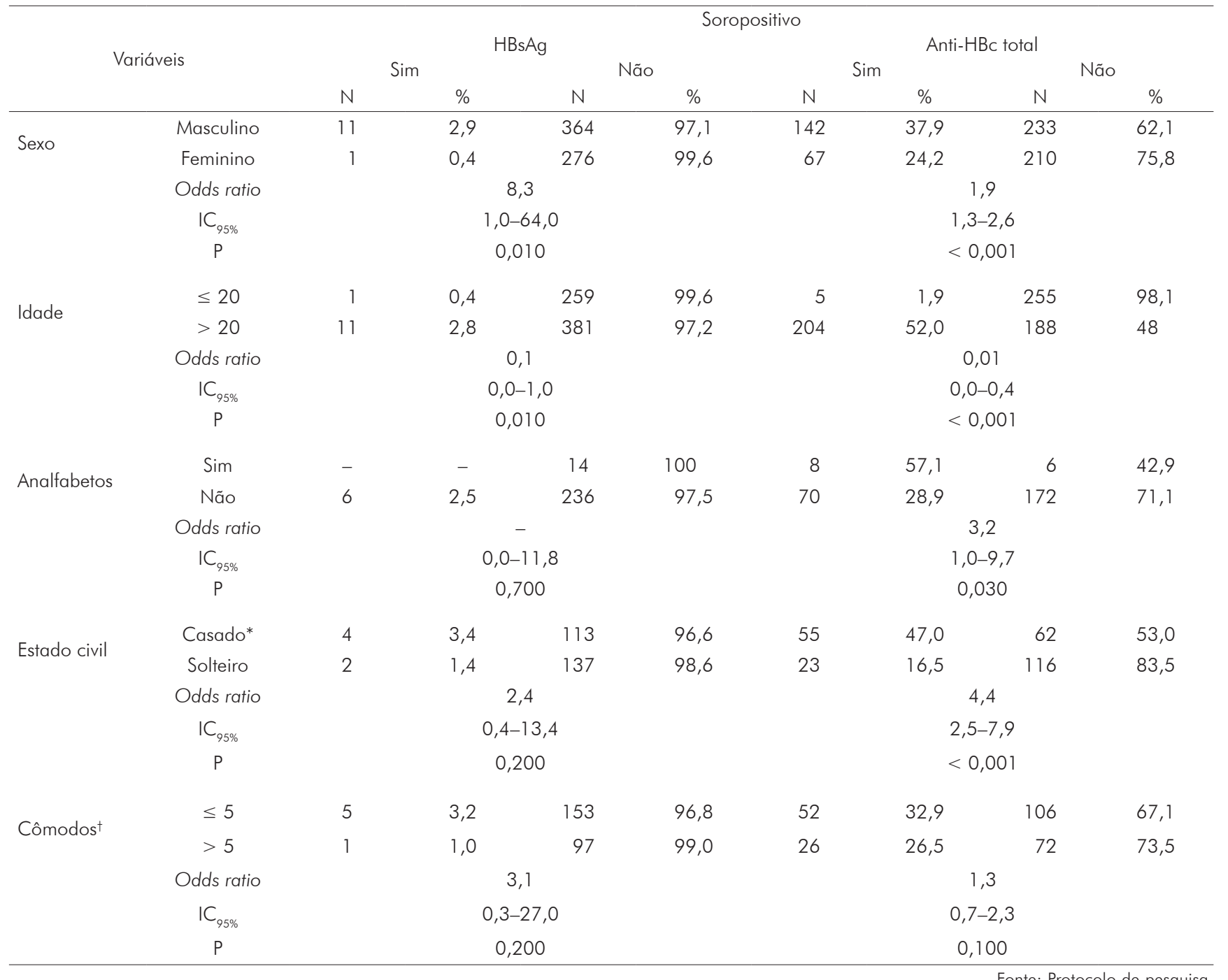

N. Número de indivíduos; IC : Intervalo de confiança 95\%; P. p-valor. Analfabeto: Sem escolaridade/sabe assinar o nome** $*$ incluem-se Osotocolo de pesquisa. viúvos e divorciados; ${ }^{\dagger}$ Número de cômodos na residência.

Sinal convencional utilizado: - Dado numérico igual a zero não resultante de arredondamento. 
A análise das características sociodemográficas, obtidas pela aplicação dos QEl, foi baseada em 256 fichas respondidas. Destas, 50,3\% relataram viver em casas construídas em alvenaria; $62,1 \%$ em residências que continham de um a cinco cômodos; $76,9 \%$ possuíam banheiro no interior da casa, sendo que desse total, 94,1\% eram fossas sépticas; 91,0\% informaram que as casas eram abastecidas com água de poço comum; e 100\% tinham acesso à energia elétrica.

Para anti-HBc total (Tabela 4) verificou-se que as variáveis "analfabetos" e "estado civil" (casados, viúvos, divorciados e separados) estiveram associadas ao risco de infecção pelo $\operatorname{VHB}(p<0,05)$. Não houve associação entre $\mathrm{HBsAg}$ e variáveis sociodemográficas $(p>0,05)$.

\section{VARIÁVEIS EPIDEMIOLÓGICAS}

Dos 660 questionários epidemiológicos aplicados, foram obtidas 256 fichas respondidas. Para os fatores de risco que favorecem a transmissão do tipo parenteral, pode-se destacar a extração dentária ( $\mathrm{n}=199 ; 77,7 \%$ ), histórico de cirurgia ( $\mathrm{n}=94$; $36,7 \%)$, transfusão sanguínea ( $\mathrm{n}=30 ; 11,7 \%$ ), tatuagem e/ou piercing $(n=19 ; 7,0 \%)$ e injeção por curioso ( $\mathrm{n}=15 ; 5,9 \%)$. $\bigcirc$ compartilhamento de materiais de higiene pessoal foi relatado por mais da metade dos indivíduos entrevistados ( $n=138 ; 53,9 \%$ ). Dentre os itens, o alicate de unha foi o mais citado ( $\mathrm{n}=110 ; 42,9 \%$ ), seguido de tesoura de unha ( $n=11 ; 4,3 \%)$, aparelho de barbear ( $n=10 ; 3,9 \%)$ e escovas de dente ( $n=7 ; 2,8 \%)$.

$\mathrm{Na}$ análise entre resultado reagente para $\mathrm{HBsAg}$ e os fatores de risco não foi observada nenhuma associação estatística significativa. $\mathrm{Na}$ análise univariada de possíveis associações entre um resultado anti-HBc total "positivo" e os fatores de risco, verificou-se que relato de cirurgia anterior, exodontia, injeção por curioso, compartilhamento de materiais de higiene pessoal e a não utilização preservativos exibiram resultados estatisticamente significantes $(p<0,05)$.

\section{DISCUSSÃO}

Com características ribeirinhas, as localidades de Cachoeira do Teotônio e Vila Amazonas foram diretamente atingidas pelos impactos provenientes da construção da UHE de Santo Antônio, em função da elevação do nível d'água do reservatório. $\bigcirc$ remanejamento dessas famílias para outros locais foi, sem dúvida, o motivo que dificultou a aplicação do QEF e do QEl em toda a população envolvida no projeto original (660 indivíduos). Entretanto, aproximadamente $39 \%$ da população retornou 0 questionário respondido. Esse valor foi considerado representativo para o presente estudo.

No início dos anos 2000, algumas regiões do Norte do Brasil eram tidas como de alta prevalência de infecções pelo VHB, quando comparadas com outras regiões brasileiras ${ }^{11}$. A soroprevalência direcionava para uma taxa geral em torno de $10 \%$ para anti-HBc total. $\bigcirc$ presente estudo mostrou uma taxa geral de anti-HBc total positivo de 32,1\%. Considerando - Brasil, em 2010 foram registrados 13.188 casos confirmados de hepatite $B$, sendo que a Região Norte respondeu por $13,3 \%{ }^{5}$ e, destes, Rondônia representou $19,7 \%$.

Sabe-se que a transmissão da hepatite B entre moradores locais e migrantes pode ocorrer com uma frequência maior na área rural ${ }^{12}$. Desde 2008, o Estado de Rondônia tem passado por um período de transição no aspecto econômico e demográfico, decorrente da construção de duas grandes UHE no rio Madeira, no Município de Porto Velho. Estima-se que esses empreendimentos tenham estimulado a migração de mais de 100 mil pessoas, direta e/ou indiretamente ligadas a essas obras civis, distribuídas entre a área urbana e rural de Porto Velho. Observando-se que - contato sexual é uma das vias de transmissão da hepatite B, o risco do aumento da incidência é considerável.

No Brasil, a distribuição do VHB não é homogênea e a Amazônia brasileira pode apresentar todos os três padrões de endemicidade: baixa, alta e intermediária ${ }^{13}$. Estudos realizados nesta Região apontam para percentuais de portadores crônicos do VHB entre 3,3\% e 4,8\% $14,15,16$. Outros estudos realizados em Rondônia apontam para prevalências entre 6,7\% e 35,3\%17,18. Mesmo sendo razoavelmente menor a proporção do presente estudo, não se pode ignorar a presença do VHB em área de intensa concentração humana ocasionada por ondas migratórias, temporárias ou não, principalmente se considerada a elevada taxa de detecção apresentada anteriormente ${ }^{5}$.

A multiciplidade de parceiros sexuais sem 0 uso de preservativos, a homossexualidade, o uso de drogas, o compartilhamento de lâminas de barbear, também são fatores de transmissão que reforçam essa associação 19,20. Já a transmissão vertical, que representa o maior meio de transmissão do VHB em regiões onde a prevalência do $\mathrm{VHB}$ é alta ${ }^{21}$, não demonstra ser muito frequente entre os indivíduos analisados no presente estudo.

A transmissão horizontal costuma ser comum durante a infância por meio do contato interpessoal ou por líquidos corporais que contém o $\mathrm{VHB}$ em regiões de alta endemicidade 22 . De acordo com a Organização Mundial da Saúde', nas áreas de baixa endemicidade, adolescentes e adultos jovens são alvos primários na transmissão do $\mathrm{VHB}$, enquanto que nas áreas de média endemicidade, todas as faixas etárias estão englobadas de modo variável, tendo como influências os aspectos culturais e socioeconômicos.

No Brasil, o Programa Nacional de Imunizações foi criado em 1973. Em 1993, foram criados os Centros de Referência para Imunobiológicos Especiais (Cries). 
Em 1996, ampliaram a oferta de vacinas para todas as crianças abaixo de 1 ano de idade, com ênfase em recém-nascidos ${ }^{23}$. Os resultados mostram que a prevalência global de indivíduos com o marcador sorológico anti-HBc total reagente $(32,1 \%)$ é quatro vezes superior à taxa brasileira ${ }^{11}$. Os grupos etários que apresentaram baixa prevalência deste marcador sorológico foram os mais jovens (0-21 anos de idade). Entretanto, este mesmo grupo apresentou as maiores frequências de vacinados e suscetíveis. Isso demonstra que a cobertura vacinal não está atingindo seu objetivo nesta área e que pode apresentar razões diversas ${ }^{24}$, como oportunidade perdida de vacinação e falta de conhecimento acerca de vacina. Situação similar é relatada em estudo na Amazônia Legal14,25. Em contraste, quando devidamente implantado o esquema vacinal, observa-se uma redução significativa do VHB nos menores de 5 anos de idade ${ }^{26}$.

Por outro lado, o aumento do anti-HBc total a partir do grupo etário 16-21 anos sugere a importância da transmissão sexual, pois o surgimento de indivíduos infectados pelo VHB a partir deste grupo etário é coerente com o padrão de transmissão encontrado em áreas de baixa endemicidade ${ }^{27}$. Sendo assim, a ocorrência de portadores do VHB a partir desse grupo etário no presente estudo e associada à transmissão horizontal, pode estar favorecendo a circulação do vírus, como citado na literatura ${ }^{28,29}$, sugerindo uma redução na expectativa de vida e elevação do número de óbitos por hepatite $B$ e hepatocarcinoma associado ao VHB registrados no Brasil na última década ${ }^{30}$.

A elevada prevalência de uso compartilhado de objeto e/ou utensílio de higiene pessoal é outro fator que favorece a transmissão na área do presente estudo, condizente com relatos anteriores ${ }^{28,29}$. A exodontia também é um importante mecanismo que pode disseminar $\circ \mathrm{VHB}^{31}$. A transfusão sanguínea não apresentou associação com o HBsAg. Isso se deve a melhorias no controle de qualidade dos bancos de sangue observados no Brasil e países vizinhos ${ }^{32,33}$.

Outros fatores socioeconômicos, o analfabetismo e estado civil, apresentaram associação com o VHB. Isso pode ser explicado pela falta de conhecimento sobre os meios de transmissão do VHB e da conduta de multiplicidade de parceiros sexuais sem mecanismos de proteção.

\section{CONCLUSÃO}

Embora os dados encontrados no presente estudo não possam ser generalizados para todo o Estado, eles são úteis para avaliar a situação epidemiológica da hepatite $B$ na região do rio Madeira. Com o aumento populacional demandado pelas obras civis das UHE em Rondônia, o potencial de transmissão não pode ser desconsiderado. A baixa cobertura vacinal e grande porcentagem de indivíduos suscetíveis observadas são preocupantes.

Além de uma campanha vacinal mais abrangente e um trabalho de educação em saúde dinâmico e permanente, faz-se necessária a realização de estudos soroepidemiológicos mais amplos a respeito do VHB, que contemplem a análise dos fatores de transmissão envolvidos na disseminação desse vírus. Desta forma, o entendimento mais profundo da epidemiologia da doença permitirá fornecer informações que podem reforçar as tomadas de decisões de forma global e mais efetiva no Estado, principalmente em relação ao controle da doença, bem como a conscientização por parte da população quanto aos métodos de proteção.

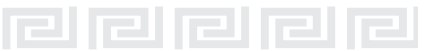

\section{Serumepidemiological profile of hepatitis B in riverine localities of the Madeira River, Porto Velho, Rondônia State, Brazil}

\begin{abstract}
It was conducted an observational study in two villages in Porto Velho, Rondônia State, Brazil, in order to evaluate the seroepidemiological profile of hepatitis B virus (HBV) and the risk factors in its transmission. Six hundred sixty samples were analyzed by ELISA method for serological tests for HBV (anti-HBc total, anti-HBs and HBsAg). The results showed that $50.7 \%$ (335) of samples were positive for some kind of serological marker. HBsAg was positive in 12 (1.8\%) samples, 11 (91.6\%) were from male ones. Anti-HBc total was positive in 212 (32.1\%) samples distributed in all groups, except for the range of 11 to 16 years old. Anti-HBs was positive in 239 (36.2\%) samples with the highest prevalence in groups over 21 years old. This study showed that the analyzed locations have low endemicity for chronic HBV carriers, however, there was a high prevalence of infection. The percentage of susceptible individuals was 49.2\% (325), the highest was in individuals between 6 to 15 years old. Aspects of transmission such as dental extractions, surgeries and sharing personal hygiene materials are possibly associated with HBV infection in the analyzed region. Furthermore, the low percentage of anti-HBs marker indicates an immunization coverage failure in the population of this study.
\end{abstract}

Keywords: Hepatitis B virus; Prevalence; Rural Population; Immunization Coverage. 


\section{Perfil seroepidemiológico de la hepatitis B en localidades ribereñas del río Madeira, en Porto Velho, Estado de Rondônia, Brasil}

\section{RESUMEN}

En dos localidades ribereñas de Porto Velho, Estado de Rondônia, Brasil, se realizó un estudio transversal de observación para evaluar el perfil seroepidemiológico del virus de la hepatitis B (VHB) y los posibles factores de riesgo para la transmisión. Se analizaron 660 muestras por ELISA realizando pruebas serológicas de VHB (anti-HBc total, anti-HBs y HBsAg). Los resultados mostraron que el 50,7\% (335) de las muestras fueron positivas para algún tipo de marcador serológico. HBsAg fue positivo en $12(1,8 \%)$ muestras, $11(91,6 \%)$ eran hombres. Anti-HBc total reactivo ocurrió en $212(32,1 \%)$ muestras y se distribuye en todos los grupos de edad, excepto la franja entre los 11 y los 16 años de edad. El reactivo anti-HBs estuvo presente en 239 (36,2\%) muestras, con mayor prevalencia en los grupos de edad a partir de los 21 años. Este estudio mostró que las localidades analizadas tienen una baja endemicidad para portadores crónicos, sin embargo, se observó alta prevalencia de la infección. El porcentaje de individuos susceptibles fue de 49,2\% (325), siendo mayor entre las personas de 6 a 15 años de edad. Los factores de transmisión, tales como extracciones dentales, cirugías y el compartido de materiales de higiene personal, están posiblemente asociados a la infección por el VHB en la región analizada. Además, el bajo porcentaje del marcador anti-HBs lo dirige a un fallo en la cobertura de vacunación en la población de este estudio.

Palabras clave: Virus de la Hepatitis B; Prevalencia; Población Rural; Cobertura de Vacunación.

\section{REFERÊNCIAS}

1 World Health Organization. Hepatitis B [Internet]. Geneva: World Health Organization; 2014 [cited 2014 Jan 10]. (Fact sheet; no. 204). Available from: http://www.who.int/mediacentre/factsheets/ fs204/en/.

2 Lavanchy D. Hepatitis B virus epidemiology, disease burden, treatment, and current and emerging prevention and control measures. J Viral Hepat. 2004 Mar; 11 (2):97-107.

3 Shepard CW, Simard EP, Finelli L, Fiore AE, Bell BP. Hepatitis B virus infection: epidemiology and vaccination. Epidemiol Rev. 2006 Jun;28(1):11225

4 Ministério da Saúde (BR). Secretaria de Vigilância em Saúde. Departamento de Vigilância Epidemiológica. Hepatites virais: o Brasil está atento. 3. ed. Brasília: Ministério da Saúde; 2008. 60 p. (Série B: Textos básicos de saúde).

5 Ministério da Saúde (BR). Secretaria de Vigilância Epidemiológica. Departamento de DST, Aids e Hepatites Virais. Bol Epidemiol Hepatites Virais. 2012 jul 25;3(1):1-172.

6 Instituto Brasileiro de Geografia e Estatística. Estados@: Rondônia - censo demográfico 2010 [Internet]. Rio de Janeiro: IBGE; 2010 [citado 2011 out 1]. Disponível em: http://www.ibge.gov.br/ estadosat/perfil.php? sigla $=$ ro.

7 Katsuragawa TH, Cunha RPA, Souza DCA, Gil LHS, Cruz RBM, Silva AA, et al. Malária e aspectos hematológicos em moradores da área de influência dos futuros reservatórios das hidrelétricas de Santo Antônio e Jirau, Rondônia, Brasil. Cad Saude Publica. 2009 jul;25(7): 1486-92.
8 Katsuragawa TH, Gil LHS, Tada MS, Silva AA, Costa JDN, Araujo MS, et al. The dynamics of transmission and spatial distribution of malaria in riverside areas of Porto Velho, Rondônia, in the Amazon Region of Brazil. PLoS One. 2010 Feb;5(2):e9245.

9 Katsuragawa TH, Gil LHS, Lima AA, Freitag EM, Santos TM, Nascimento Filha MT, et al. Selective intermittent preventive treatment of vivax malaria: reduction of malaria incidence in an open cohort study in Brazilian Amazon. Malar Res Treat. 2013;2013(ID310246): 1- 11.

10 Mast EE, Weinbaum CM, Fiore AE, Alter MJ, Bell $B P$, Finelli $L$, et al. A comprehensive immunization strategy to eliminate transmission of hepatitis $B$ virus infection in the United States: recommendations of the Advisory Committee on Immunization Practices (ACIP) part II: immunization of adults. MMWR Recomm Rep. 2006 Dec;55(RR16):1-25.

11 Ferreira CT, Silveira TR. Hepatites virais: aspectos da epidemiologia e da prevenção. Rev Bras Epidemiol. $2004 \mathrm{Dec} ; 7(4): 473-87$.

12 Passos AD, Gomes UA, Figueiredo JF, Nascimento MM, Oliveira JM, Gaspar AM, et al. Influência da migração na prevalência de marcadores sorológicos de hepatite $B$ em comunidade rural: 1 - análise da prevalência segundo local de nascimento. Rev Saude Publica. 1993 fev;27(1):305.

13 Ministério da Saúde (BR). Secretaria de Vigilância em Saúde. Departamento de DST, Aids e Hepatites Virais. $A B C D E$ do diagnóstico para as hepatites virais. Brasília: Ministério da Saúde; 2009. 24 p. (Série A. Normas e manuais técnicos). 
14 Souto FJD, Fontes CJF, Oliveira SS, Yonamine F, Santos DRL, Gaspar AMC. Prevalência da hepatite $B$ em área rural de município hiperendêmico na Amazônia Mato-Grossense: situação epidemiológia. Epidemiol Serv Saude. 2004 jun;13(2):93-102.

15 Khouri ME, Duarte LS, Ribeiro RB, Silva LFF, Camargo LMA, Santos VA, et al. Seroprevalence of hepatitis B virus and hepatitis C virus in Monte Negro in the Brazilian Western Amazon Region. Clinics. 2005 Jan-Feb;60(1):29-36.

16 Oliveira CF, Silva AV, Santos KN, Fecury AA, Almeida MK, Fernandes AP, et al. Hepatitis B and $C$ virus infection among Brazilian Amazon riparians. Rev Soc Bras Med Trop. 2011 SepOct;44(5):546-50.

17 Ferrari JO, Ferreira MU, Tanaka A, Mizokami $M$. The seroprevalence of hepatitis $B$ and $C$ in an Amerindian population in the southwestern Brazilian Amazon. Rev Soc Bras Med Trop. 1999 MayJun;32(3):299-302.

18 Katsuragawa TH, Cunha RPA, Villalobos Salcedo JM, Souza DCA, Oliveira KRV, Gil LHS, et al. Alta soroprevalência dos marcadores das hepatites B e C na região do alto rio Madeira, Porto Velho, Rondônia, Brasil. Rev Pan-Amaz Saude. 2010 jun; 1 (2):93-8.

19 Alter MJ. Heterosexual transmission of hepatitis B and implication for vaccine: prevention strategies. In: Bennet DL, editor. The control of hepatitis B: the role of prevention in adolescence. London: Gower Medical; 1991. p. 21-5.

20. Tura FLR, Madeira CM, Silva AO, Gaze R, Carvalho DM. Representações sociais de hepatite e profissionais de saúde: contribuições para um (re) pensar da formação. Cienc Cuid Saude. 2008 abrjun; $7(2): 207-15$.

21 Darani M, Gerber M. Hepatitis B antigen in vaginal secretions. Lancet. 1974 Oct;304(7887):1008.

22 Silveira TR, Cunha J, Krebs LS, Ramalho L. Avaliação do grau de conhecimento e de proteção de ginecologistas e obstetras do Rio Grande do Sul em relação à hepatite. Rev AMRIGS. 2003 julset;47(3): 193-201.

23 Ministério da Saúde (BR). Secretaria de Vigilância em Saúde. Programa Nacional de Imunizações: 30 anos. Brasília: Ministério da Saúde; 2003. 208 p. (Série C: Projetos e programas e relatórios).
24 Carvalho AMC, Araujo TME. Fatores associados à cobertura vacinal em adolescentes. Acta Paul Enferm. 2010;23(6):796-802.

25 Souto FJD, Ozaki KS, Pereira ES, Sousa KM, Silveira WN, Pacheco MS. Efeito da vacina contra hepatite $B$ em indivíduos com anticorpos contra o antígeno central da hepatite B (anti-HBc) como único marcador. Gastroenterol Endosc Digest. $2001 ; 20(2): 43-7$.

26 Braga WSM, Brasil LM, Souza RAB, Melo MS, Rosas MDG, Castilho MC, et al. Prevalência da infecção pelo vírus da hepatite $\mathrm{B}(\mathrm{VHB})$ e da hepatite Delta (VHD) em Lábrea, Rio Purus, Estado do Amazonas. Epidemiol Serv Saude. 2004 mar;13(1):35-46.

27 Silveira TR, Fonseca JC, Rivera L, Fay OH, Tapia R, Santos Jl, et al. Hepatitis B seroprevalence in Latin America. Rev Panam Salud Publica. 1999 Dec;6(6):378-83.

28 Lobato C, Tavares-Neto J, Rios-Leite M, Trepo C, Vitvitski L, Parvaz P, et al. Intrafamilial prevalence of hepatitis B virus in Western Brazilian Amazon region: epidemiologic and biomolecular study. J Gastroenterol Hepatol. 2006 May;21 (5):863-8.

29 Brasil LM, Fonseca JCF, Souza RB, Braga WSM, Toledo LM. Prevalência de marcadores para o vírus da hepatite B em contatos domiciliares no Estado do Amazonas. Rev Soc Bras Med Trop. 2003 setout;36(5):565-70.

30 Tauil MC, Amorim TR, Pereira GF, Araújo WN. Mortalidade por hepatite viral B no Brasil, 20002009. Cad Saude Publica. 2012 mar;28(3):472-8.

31 Hyams KC. Mosquito transmission of hepatitis B. Trop Geogr Med. 1989 Jul;41 (3):185-9.

32 Wanderley DM, Gonzales TT, Pereira MS, Nascimento RD, Moraes-Souza H. Controle da hemoterapia e da doença de Chagas transfusional: 1988 e 1990. Rev Saude Publica. 1993 dez;27(6):430-5.

33 Beltrán M, Berrío-Pérez M, Bermúdez MI, ReyBenito G, Camacho B, Forero P, et al. Detección de hepatitis B oculta em donantes de bancos de sangre, Colombia 2008-2009. Biomedica. $2011 ; 31(4): 580-9$.

Recebido em / Received / Recibido en: 11/4/2014 Aceito em / Accepted / Aceito en: 6/2/2015 